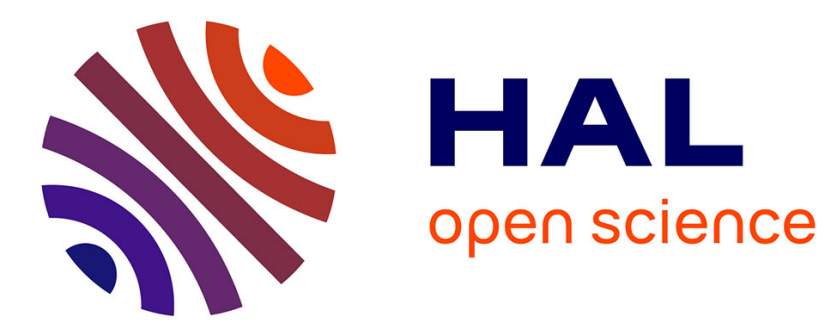

\title{
Computational Cost Efficient Model of Losses for Multi-port Active-bridge Converters
}

Soleiman Galeshi, David Frey, Yves Lembeye

\section{To cite this version:}

Soleiman Galeshi, David Frey, Yves Lembeye. Computational Cost Efficient Model of Losses for Multiport Active-bridge Converters. IEEE Energy Conversion Congress \& Expo - ECCE 2020, Oct 2020, Détroit (virtual), United States. 10.1109/ECCE44975.2020.9236119 . hal-03145561

\section{HAL Id: hal-03145561 \\ https://hal.science/hal-03145561}

Submitted on 18 Feb 2021

HAL is a multi-disciplinary open access archive for the deposit and dissemination of scientific research documents, whether they are published or not. The documents may come from teaching and research institutions in France or abroad, or from public or private research centers.
L'archive ouverte pluridisciplinaire HAL, est destinée au dépôt et à la diffusion de documents scientifiques de niveau recherche, publiés ou non, émanant des établissements d'enseignement et de recherche français ou étrangers, des laboratoires publics ou privés. 


\section{Computational Cost Efficient Model of Losses for Multi-port Active-bridge Converters}

\author{
Soleiman Galeshi \\ UNIV. GRENOBLE ALPES, CNRS \\ GRENOBLE INP*, G2ELAB \\ Grenoble, France \\ soleiman.galeshi- \\ mooziraji@g2elab.grenoble-inp.fr
}

\author{
David Frey \\ UNIV. GRENOBLE ALPES, CNRS \\ GRENOBLE INP*, G2ELAB \\ Grenoble, France \\ david.frey@g2elab.grenoble-inp.fr
}

\author{
Yves Lembeye \\ UNIV. GRENOBLE ALPES, CNRS \\ GRENOBLE INP*, G2ELAB \\ Grenoble, France \\ yves.lembeye@g2elab.grenoble-inp.fr
}

\begin{abstract}
Multi-port active-bridge (MAB) converters, in single and clustered architecture, are used for many different applications. Due to intertwining of currents and powers of all their ports, finding a closed form solution for optimal control of single and clustered MAB converters that leads to highest efficiency is not an option. Therefore, optimization of power distribution in a cluster of MAB converters must be done by trial and error, which requires a model that can predict the losses. A detailed and accurate model of losses, however, would need large amounts of memory and processing resources, which makes real-time optimization of control and modulations costly, if not almost impossible. This work seeks to propose a computational cost efficient model, which is able to predict the losses with acceptable accuracy. Given the operating point of a MAB converter, this model is able to predict total losses using polynomial calculations. This efficient model can be employed for optimizing control of a cluster of MAB converters. Predictions of this model are compared against experimental measurements to validate its correct operation.
\end{abstract}

Keywords-component, formatting, style, styling, insert (key words)

\section{INTRODUCTION}

Multi-port active-bridge (MAB) converters are finding their place in many different areas of industry because of their advantages, such as intrinsic galvanic isolation, soft switching, small passive components and bidirectional power transfer between multiple resources. They can be employed to transfer energy between generation, storage and consumption units; these three units can be in an electric car/aircraft [1] or in a green building/micro-grid [2]. Their modular structure makes them a good candidate for distributed converter systems, where a cluster of converters work together to perform certain functions. They can be used in series/parallel to connect HVAC networks to dc networks [3].

A power routing algorithm is proposed in [4] that tries to balance lifetime of parallel cascaded $\mathrm{H}$-bridge and dual activebridge (DAB) converters based on junction temperature. It should be noted that MAB converters usually operate in soft switching condition and their switching losses are very small. Therefore, junction temperature cannot be a proper indicator of the total losses. Parallel operation of DAB converters connected for energy collection of wind farms is studied in [5]. The main goal in designing the controller in [5] is dynamic performance and stability. Employing a model of losses allows adding efficiency to the design optimization goals. Application of clustered MAB converters in smart buildings has been studied in [2], however, no optimization strategy is proposed for routing power between the converters. Several other works in the literature study control and power routing of parallel or clustered active-bridge converters, however they do not pursue optimal power distribution between them.

A general model of losses can be used to determine optimal power distribution in a cluster of MAB converters [6 PCIM]. Many works have studied losses in DAB converters. They propose analytical calculation for each type of losses in a DAB converter, including core losses, conductions losses, and switching losses. When determining losses in a DAB converter, only one phase shift can be defined. This phase shift easily gives the power and the current that flow between the two ports. The operating point parameters (voltages and currents) that are required for calculation of losses can be determined using polynomial equations in this case. However, in case of an $n$-port MAB converter, $(n(n-1)) / 2$ phase shifts can be defined between each pair of ports. There are $(n(n-1)) / 2$ power transfers between each pair of ports, too. The winding voltages and currents are affected by all the phase shifts at the same time. The shapes of transformer voltage and the currents get more and more complex and difficult to predict with an increasing number of ports. It will still be possible to calculate the losses in a MAB converter, but the number of calculations quickly increases. In case of a cluster of MAB converters, as described in $[2,4]$, the optimization process would become even more difficult.

This work seeks to present a simple, but accurate enough model for losses. This model can be employed in finding optimized modulation of a MAB converter and optimal power distribution in a network of clustered MAB converters. In the following sections, an overview of the losses in a MAB converter will be presented. The approximations that help reach the simplified model will then be explained. The final section presents experimental validation of the model.

\section{LOSSE IN ACTIVE-BRIDGE CONVERTERS}

A quad active-bridge (QAB) converter is illustrated in Fig. 1. This converter has four active-bridges, four inductors, and a four-winding transformer. The four active-bridges generate square-wave voltages with phase shifts between them. The phase shifts lead to flow of power between the ports. In this regard, the desired power transfer between the ports, are the inputs of the converter control system and the phase shift are the outputs. The model should be able to determine losses in the converter, based on the powers or their corresponding phase shifts.

Major types of losses in MAB converters are switching losses; conduction losses in the switches and windings of the inductor and the transformer; and, iron losses in magnetic cores of the inductors and the transformer. In order to assess the switching losses, voltages and currents at the switching

* Institute of Engineering Univ. Grenoble Alpes 


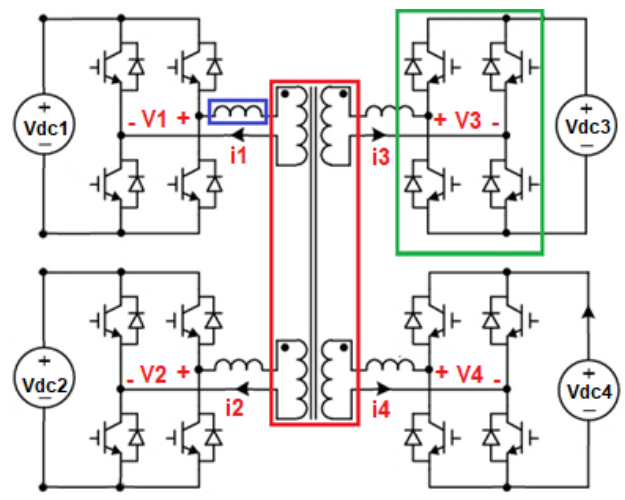

Fig. 1. A quad active-bridge converter with four active-bridges (the green box) and inductors (the blue box) and a four-winding transformer (the red box).

moments are needed. Effective current value is the important parameter that should be determined to predict conduction losses. Core losses depend on shape and magnitude of magnetic flux density. Magnetic flux density itself can be determined from shape and magnitude of voltage across the windings on the core.

\section{APPROXIMATION OF THE LOSSES}

\section{A. Scope of Application and Assumptions}

Optimization in power electronic converters corresponds to various aspects, such as sizing magnetic components and semiconductors, thermal analysis, cooling system design, and switching modulations. These optimizations are performed through different approaches. The first approach is to determine the optimization goal as a function of input parameters and find the optimum using mathematical analysis. As systems are getting more and more complex, this approach is not always possible, i.e., it is not possible to determine a closed form solutions that gives the optimum point in big and complex systems. Numerical approaches, such as finite element analysis in thermal design, are able to find the optimum point whenever the analytical approach fails. The main drawback of numerical approaches is that they require large memory and processing resources and take a long time to find the optimum point. It means that numerical approaches are not the proper solution in real-time optimization and online control.

In cases where analytical and numerical approaches are not an appropriate choice for optimization, reduced order models (ROM) can be helpful. With the current trend of emerging more and more complex architectures in power electronics, ROM is becoming more relevant and useful in both academic and industrial applications [7-9]. Reference [7] presents a ROM of and induction generator for fixed speed wind turbines. This model can be used in design and control of the generators. A ROM of modular multilevel converters (MMC) is presented in [8]. MMC is a clear example of large power electronic systems where ROM can be a handy tool. Reference [9] studies a ROM for a network of arbitrary number of parallel single-phase converters. Considering the internal phenomenon of each converter and the possible dynamic interaction between the converters, the state and solution spaces are large. Therefore, analytical and numerical approaches will not be appropriate for optimization. The ROM in [9] can be useful in dynamic analysis and control of parallel converters.
The goal of this work is to propose a ROM for MAB converters that can be used for control and optimization purposes, including optimal power flow in a cluster of MAB converters and internal control of a single converter. It should be noted that soft switching conditions in MAB converters allows operation with negligible losses at tens and hundreds of kilohertz. Considering the high frequency, switching losses would be too high if the control system was not able to maintain soft switching conditions. Therefore, it is assumed that MAB converters rarely operate at hard switching conditions and it is simpler to ignore the switching losses. It should be mentioned that one of the goals of clustering is to employ the converters near their nominal power, where their efficiency is high and soft switching conditions are met. Detailed switching process and calculation of soft and switching losses in MAB converters is presented in [6].

Conduction losses have a direct relationship with resistance of transformer and inductor windings. These resistances can be analytically calculated for each converter based on the length and cross section area of the wires, in addition to some geometric parameters such as core size and winding fitting coefficients. However, the additional losses due to skin effect and eddy losses (because of proximity effect) cannot be ignored. Several works propose methods of calculation these effects, but doing so will add to complexity and computational cost of the ROM. In this regard, it is assumed that resistances of the windings are measured using an impedance analyzer at the switching frequency. On-state resistance of mosfet channel vary with operating conditions, too, but these variations are usually negligible compared to winding resistances. Hence, datasheet value of on-state resistance of the channels at designed gate-source voltage, nominal current and $75{ }^{\circ} \mathrm{C}$ is used in the model. Considering these assumptions, determining effective current magnitudes will be enough for prediction of conduction losses.

The ROM in this work is a series of mathematical calculations that gives an estimation of the losses in a MAB converter based on its design parameters, such as winding resistances, size of magnetic cores and dc link voltages, and operating point, i.e., phase shift between switchings of active bridges. Variation of parameters from their nominal value can affect the accuracy of the predictions. The extent of this impact will be studied in section IV.

\section{B. Conduction Losses}

Conduction losses occur in the channels of the mosfet switches and in the windings of the transformer and the inductors. In order to determine conduction losses, effective value of the currents should be calculated. Operating parameters of a sample switching of a QAB is shown in Fig. 2. It can be considered as a general set of voltage/current waveforms of a QAB converter. The phase shifts, from the smallest to the largest, are $\varphi_{1}, \varphi_{2}, \varphi_{3}$ and $\varphi_{4}$, respectively. Based on Fig. 2, currents can be approximated as trapezoidal waveforms. The two currents that correspond to the smallest and the largest phase shifts have the same general shape (i1 and i4 in Fig. 2, respectively) and their trapezoidal approximation is shown in Fig. 3. In order to determine effective values of $i_{1}$ and $i_{4}$, their maximum value is needed. This value can be determined from

$$
i_{L k}=\frac{1}{L} \int v_{L k} d t
$$




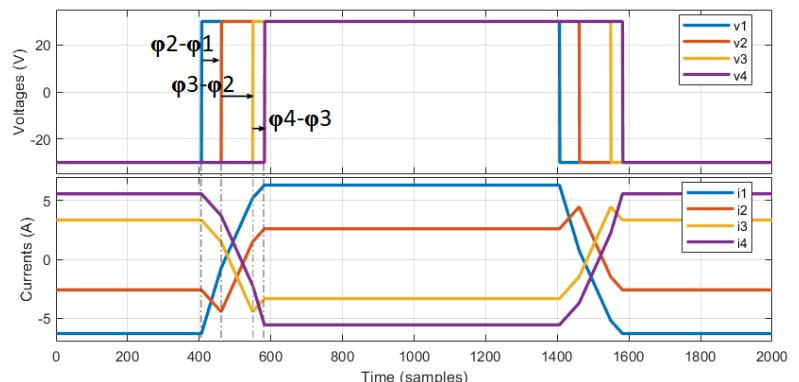

Fig. 2. General voltage and current waveforms of a quad active-bridge converter. Measurement points are indicated in Fig. 1. Each switching cycle has 2000 sampling points (horizental axis).

where, $V_{L k}$ is the voltage across the inductor connected to the $k$-th active bridge. Approximation of inductor voltage integration will be presented later. Knowing the maximum value of a trapezoidal waveform, its effective value can be determined from

$$
I_{M} \sqrt{\frac{1}{\pi}\left(\pi-\frac{4}{3} \varphi\right)}
$$

where, $I_{M}$ is the maximum value of the current, and $\varphi=\varphi_{4}-$ $\varphi_{1}$.

The shape of the currents that correspond to the second and the third phase shifts, $i_{2}$ and $i_{3}$ is different from $i_{1}$ and $i_{4}$. It is still possible to approximate their shape with a trapezoidal waveform, but with a slightly different approach. Fig. 4 displays three possible trapezoidal approximations of $i_{2}$. Similar approximations are possible for $i_{3}$. The effective current values resulted from these approximations were compared to effective value of the original waveform for different sets of phase shifts. The results showed that the approximation illustrated in green dotted line has maximum $3 \%$ error, while the dark blue and light blue had $+30 \%$ and $\pm 8 \%$ maximum errors, respectively. The maximum value of the approximation in green dotted line in Fig. 4 can be determined from inductor voltages (Fig. 5), and their effective value can be determined using Eq. (2) afterwards.

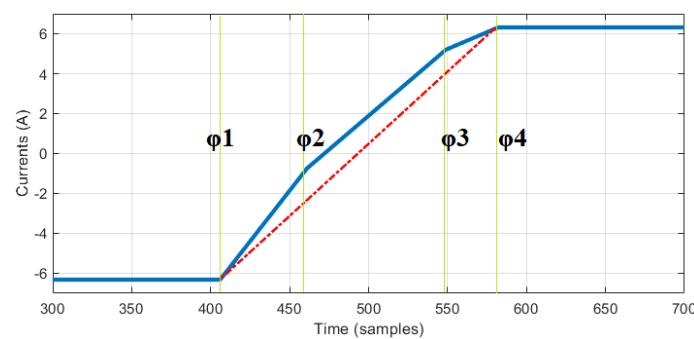

Fig. 3. Approximated waveform (red) of the current $i_{1}$ (blue) in Fig. 2. Similar approximation applies to current $i_{4}$. Vertical light green lines indicate phase shifts.

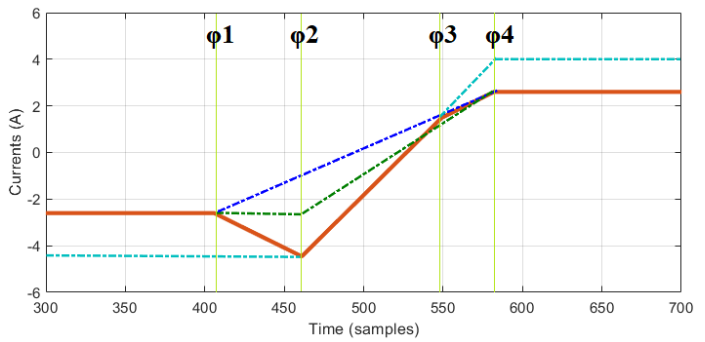

Fig. 4. Trapezoidal approximations (dark blue, light blue and dark green) of the $i_{2}$ in Fig. 2 (red). Similar approximation applies to $i_{3}$.

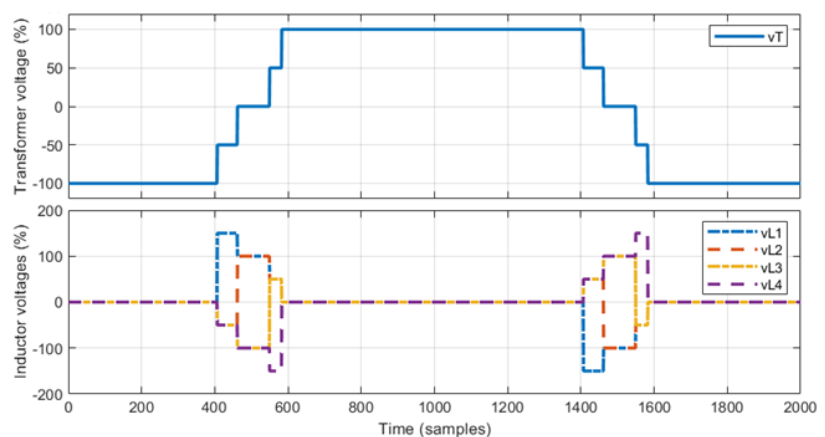

Fig. 5. The voltages across the transformer and inductor windings. Voltage units are percentage of active-bridge dc link voltages (Fig. 1).

In order to determine the shape of inductor voltages, $v_{L k}$, the voltage across transformer windings, $v_{T}$, has to be determined. Based on Fig. $1, v_{L k}=v_{k}-v_{T}, v_{k}$ being the output voltage of the $k$-th active-bridge (the square-wave voltages shown in Fig. 2). Fig. 5 shows the general shape of transformer and inductor voltages. As shown in Fig. 5, inductor voltages have steps of $\pm 150 \%$ and $\pm 50 \%$ of the dc link voltage, depending on their phase shift. Width of these steps is equivalent to phase shifts indicated in Fig. 2. Integration of inductor voltages over half a cycle can be calculated as (3) to (6). Based on (2), maximum value of currents can be predicted as $\frac{1}{2 L} \int_{0}^{\frac{1}{2 f}} V_{L}(t) d t$, or expressed as a matrix, $I_{M}$, in (7).

$$
\begin{gathered}
\int_{0}^{\frac{1}{2 f}} v_{L 1}(t) d t=\left(1.5\left(\varphi_{2}-\varphi_{1}\right)+\left(\varphi_{3}-\varphi_{2}\right)+0.5\left(\varphi_{4}-\right.\right. \\
\left.\left.\varphi_{3}\right)\right) V_{d c}
\end{gathered}
$$

$$
\int_{0}^{\frac{1}{2 f}} v_{L 2}(t) d t=\left(-0.5\left(\varphi_{2}-\varphi_{1}\right)+\left(\varphi_{3}-\varphi_{2}\right)+0.5\left(\varphi_{4}-\right.\right.
$$$$
\left.\left.\varphi_{3}\right)\right) V_{d c}
$$

$$
\int_{0}^{\frac{1}{2 f}} v_{L 3}(t) d t=\left(0.5\left(\varphi_{2}-\varphi_{1}\right)+\left(\varphi_{3}-\varphi_{2}\right)-0.5\left(\varphi_{4}-\right.\right.
$$

$\left.\left.\varphi_{3}\right)\right) V_{d c}$

$$
\begin{gathered}
\int_{0}^{\frac{1}{2 f}} v_{L 4}(t) d t=\left(0.5\left(\varphi_{2}-\varphi_{1}\right)+\left(\varphi_{3}-\varphi_{2}\right)+1.5\left(\varphi_{4}-\right.\right. \\
\left.\left.\varphi_{3}\right)\right) V_{d c}
\end{gathered}
$$

$$
I_{M}=\left[\begin{array}{l}
I_{M 1} \\
I_{M 2} \\
I_{M 3} \\
I_{M 4}
\end{array}\right]=\frac{V_{d c}}{8 L \pi f}\left[\begin{array}{cccc}
-3 & 1 & 1 & 1 \\
1 & -3 & 1 & 1 \\
-1 & -1 & 3 & -1 \\
-1 & -1 & -1 & 3
\end{array}\right] \times\left[\begin{array}{l}
\varphi_{1} \\
\varphi_{2} \\
\varphi_{3} \\
\varphi_{4}
\end{array}\right]
$$

Effective currents can now be determined as a matrix, $I_{R M S}$, using (2) and (7).

$$
I_{R M S}=I_{M} \sqrt{\frac{1}{\pi}\left(\pi-\frac{4}{3}\left[\begin{array}{cccc}
-1 & 0 & 0 & 1 \\
0 & -1 & 0 & 1 \\
-1 & 0 & 1 & 0 \\
-1 & 0 & 0 & 1
\end{array}\right] \times\left[\begin{array}{l}
\varphi_{1} \\
\varphi_{2} \\
\varphi_{3} \\
\varphi_{4}
\end{array}\right]\right)}
$$

Knowing the effective currents, measured resistances of windings and datasheet value of drain source channel on-state resistance, total conduction losses in a MAB converter can be predicted. 


\section{Core Losses}

Many works in the literature propose methods that can predict magnetic core losses. A commonly used method for this purpose is iGSE, proposed in [10]. It is method based on Steinmetz coefficients, which are determined through experimental measurements and curve fitting for different types of ferrite cores. The iGSE is well known to have accurate prediction of losses in ferrite cores when the voltage across is not sinusoidal, as in MAB converters. The main equation that gives the losses in this method is

$$
K_{i G S E} V_{c}\left(2 B_{m}\right)^{b-a} f \int_{0}^{\frac{1}{f}}|d B / d t|^{a} d t
$$

where, $K_{i G S E}, a$ and $b$ are constants, related to the core material, $V_{c}$ is the core volume, $f$ is the switching frequency, and $B$ is magnetic flux density. The two important terms that should be evaluated are $B_{m}$, maximum flux density, and $\int_{0}^{\frac{1}{f}}|d B / d t|^{a} d t$. The magnetic flux density itself can be calculated from

$$
B=\frac{\int_{0}^{\frac{1}{f}} V(t) d t}{N S}
$$

where $N$ is the number of turns, and $S$ is the cross section area of the core. Fig. 5 and (10) can help determine the two important parameters for calculations of core losses. The approach is similar to the previous section on prediction of effective currents. Maximum value of magnetic flux densities in inductor cores can be determined from (10) as in (11), and a coefficient matrix similar to the one in (7) gives the value for each inductor core.

$$
B_{\max L}=\frac{1}{2 N S} \int_{0}^{\frac{1}{2 f}} V_{L}(t) d t
$$

The second term, $\int_{0}^{\frac{1}{f}}|d B / d t|^{a} d t$, can also be calculated from Fig. 5. Based on the relationship between the voltage across the windings and the magnetic flux density in (10), $|d B / d t|=\left|\frac{V(t)}{N S}\right|$. Due to the abstract value, the coefficient matrix will be different from the one used for effective currents and maximum flux densities. Therefore, the second term can be calculated from

$$
\left(\frac{V_{d c-k}}{N_{L k} S_{L k}}\right)^{a} \frac{1}{2 \pi f}\left[\begin{array}{cccc}
-3 & 1 & 1 & 1 \\
-1 & -1 & 1 & 1 \\
-1 & -1 & 1 & 1 \\
-1 & -1 & -1 & 3
\end{array}\right] \times\left[\begin{array}{l}
\varphi_{1} \\
\varphi_{2} \\
\varphi_{3} \\
\varphi_{4}
\end{array}\right]
$$

Although the shape of the voltage across the transformer winding is different from that of the inductor windings, the same concept can still be employed to determine losses in the magnetic core of the transformer. Based on Fig. 5 and (10)

$$
B_{\max T}=\frac{V_{d c}}{8 N S \pi f}\left(2 \pi+\varphi_{1}+\varphi_{2}-\varphi_{3}-\varphi_{4}\right)
$$

and

$$
\int_{0}^{\frac{1}{f}}\left|\frac{d B_{T}}{d t}\right|^{a} d t=\left(\frac{V_{d c}}{N_{1 T} S_{T}}\right)^{a} \frac{2 \pi+\varphi_{1}+\varphi_{2}-\varphi_{3}-\varphi_{4}}{2 \pi f}
$$

\section{EXPERIMENTAL VALIDATION}

A laboratory-scale QAB converter was built in G2ELab for experimental validation of the proposed model. Fig. 6 shows the converter. Table I lists some of its details. Transformer turns are equal on every ports and so are nominal dc link voltage and inductors. This converter can transfer maximum 250 Watts on each port at $40 \mathrm{~V}$ dc link voltage and $40 \mathrm{kHz}$ switching frequency.

Validity of the model was tested at different switching frequencies and dc link voltages. A comparison between predicted and measured losses at $40 \mathrm{kHz}$ switching frequency and dc link voltage of $40 \mathrm{~V}$ is illustrated in Fig. 7 for several operating points. In this test, two ports provided power and the other two acted as loads. The horizontal axis is the total power drawn from the sources. The total input power increases from $18 \mathrm{~W}$ up to $240 \mathrm{~W}$. The difference between predicted and measured losses is indicated as "Error". Fig. 7 shows that there is a small error below $1 \mathrm{~W}$. It varies between $0.3 \mathrm{~W}$ to $0.8 \mathrm{~W}$ and its average value is $0.6 \mathrm{~W}$. This error can be due to approximations, difference between real and datasheet parameters, or accuracy of measurement devices. In any case, it shows that accuracy of the model in these operating points is in an acceptable range.

In order to verify that performance of the model remains acceptable with different dc link voltages and switching frequencies, the converter was tested with another set of operating points. The results are illustrated in Fig. 8. Dc link voltages in this test are $30 \mathrm{~V}$ and switching frequency is 20 $\mathrm{kHz}$. The maximum power is limited is this case due to reduction of dc link voltages. The error in this test is between $0.2 \mathrm{~W}$ and $0.9 \mathrm{~W}$, with average value of $0.45 \mathrm{~W}$.

TABLE I. DETAILS OF THE PROTOTYPE CONVERTER

\begin{tabular}{|c|c|}
\hline Parameter & Value \\
\hline Maximum DC voltage & $40 \mathrm{~V}$ \\
\hline Nominal power & $250 \mathrm{~W}$ each port \\
\hline External inductors & $10 \mu \mathrm{H}$ \\
\hline Transformer turns & $7: 7: 7: 7$ \\
\hline Switching frequency & $40 \mathrm{kHz}$ \\
\hline
\end{tabular}

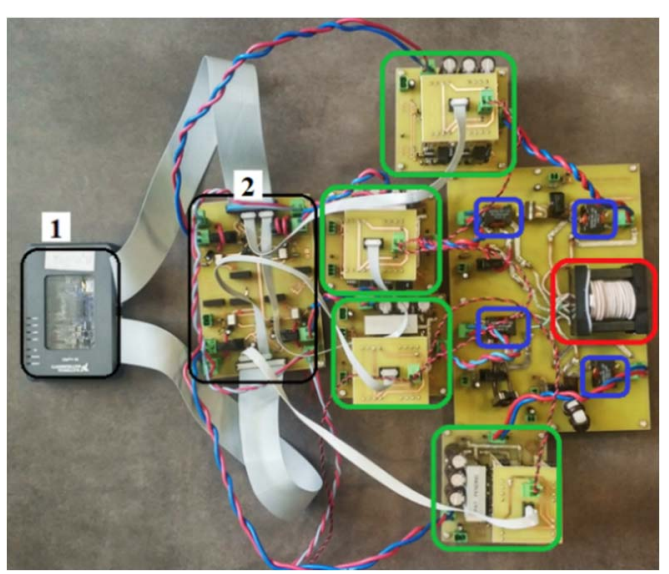

Fig. 6. The quad active-bridge converter that was built in G2ELab for experimental validation of the proposed model of losses. The boxes numbere 1 and 2 are controller and signal conditioning circuit, respectively. The green and the blue boxes indicate the four active bridges and inductors, respectively. The red box is a four-winding transformer. The colored boxes correspond to the ones in Fig. 1. 


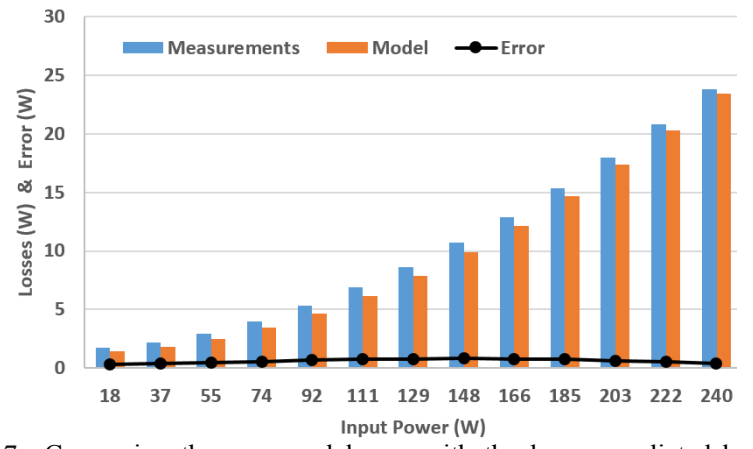

Fig. 7. Comparing the measured losses with the losses predicted by the proposed model. The switching frequency is $40 \mathrm{kHz}$ and dc link voltages are $40 \mathrm{~V}$. Efficiency of the converter is between $90 \%$ and $95 \%$.

So far, the experimental results showed that the reduced order model is able to predict the losses with acceptable accuracy. However, there are situations where this model is not able to give an accurate prediction. These situations occur when operating conditions of the converter are far from its nominal values. Fig. 9 shows the results of experiments where one of the dc link voltages is $12 \%$ above its nominal value. Dc link voltage of one the ports is $28 \mathrm{~V}$ while the rest are $25 \mathrm{~V}$. The power transfer in this test is lower than the previous tests because the de link voltages are smaller.

The results show that in low power there error is relatively large. The reason for this increase is hard switching losses, which occur at low powers when there is de link voltage variation. The reduced order model does not consider hard switching conditions and losses, hence the errors can be large when there is hard switching. As the power increases, soft switching conditions are met and switching losses become negligible, hence the error becomes smaller. However, when the power continues to increase further, circulating currents due to dc link voltage difference become large and increase the losses. The model does not currently consider losses due to dc link voltage variations and circulating currents, hence the error increases again.

\section{CONCLUSIONS}

Application of multi-port active-bridge converters is increasing in many different applications. Considering the complexity of their internal phenomenon and large number of control parameters, finding analytical methods for real-time optimization of their control is not possible. Numerical methods can be useful but require large computational and memory resources. This work presents a reduced order model with low computational costs, that can predict losses in

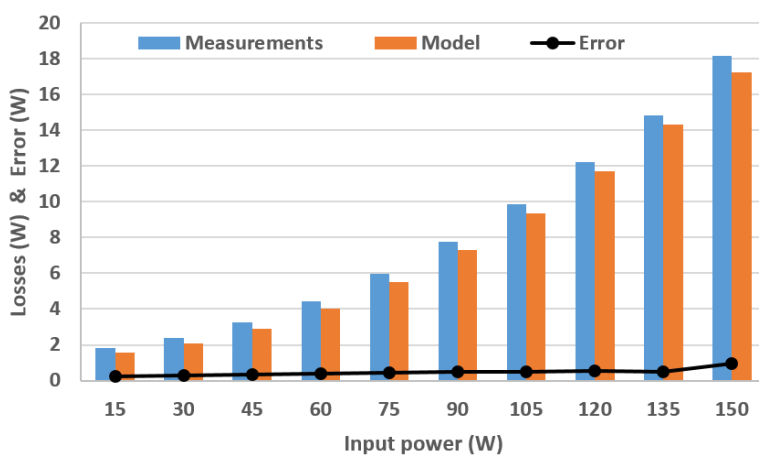

Fig. 8. Comparing the measured losses with the losses predicted by the proposed model in a different operating condition. The switching frequency is $20 \mathrm{kHz}$ and dc link voltages are $30 \mathrm{~V}$.

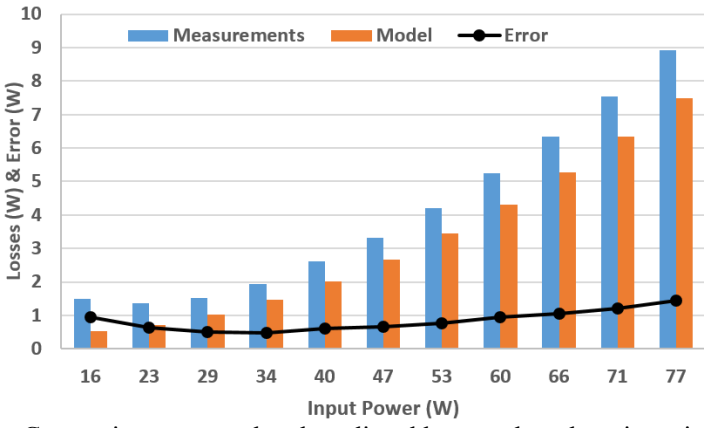

Fig. 9. Comparing measured and predicted losses when there is variation in dc link voltages. In this test, dc link voltage of all ports are $25 \mathrm{~V}$, except for one, which is $28 \mathrm{~V}$. Hard switching and circulating currents, which are not considered in the model, lead to large prediction errors.

multi-port active-bridge converters. Some approximations were used in this model to reduce the computational cost. These approximations affect accuracy of the model. Experimental tests were performed on a laboratory-scale prototype of a four-port converter. The results showed that the accuracy is acceptable if the converter works in normal operating conditions. Additional tests were performed with variation from nominal value in one of the dc links. The results showed that large variations reduce accuracy of the model, i.e., care should be given when using the model. The control system should be able to avoid situations that deteriorate the accuracy of the model.

\section{ACKNOWLEDGMENT}

This work has been partially supported by the CDP Eco-SESA receiving fund from the French National Research Agency in the framework of the "Investissements d'avenir" program (ANR-15-IDEX-02).

\section{REFERENCES}

[1] M. Blanc, Y. Lembeye, J. P. Ferrieux, C. Rizet, A. Mahe, and T. Bensalah, "Optimization of a DC/DC dual active bridge converter for aircraft application,” EPE Journal, vol. 28, pp. 182-199, October 2018.

[2] S. Galeshi, D. Frey, Y. Lembeye and D. Motte-Michellon, "Application of Clustered Multi-port Active-bridge Converters in Microgrids," 21st European Conference on Power Electronics and Applications (EPE '19 ECCE Europe), Genova, Italy, 2019.

[3] B. J. D. Vermulst, J. L. Duarte, C. G. E. Wijnands and E. A. Lomonova, "Quad-Active-Bridge Single-Stage Bidirectional Three-Phase AC-DC Converter With Isolation: Introduction and Optimized Modulation," IEEE Transactions on Power Electronics, vol. 32, pp. 2546-2557, April 2017.

[4] Y. Ko, V. Raveendran, M. Andresen and M. Liserre, "Discontinuous Modulation based Power Routing for Modular Smart Transformers," IEEE Energy Conversion Congress and Exposition (ECCE), Portland, OR, 2018, pp. 1084-1090, 2018.

[5] Y. Sang, A. Junyent-Fcrré, X. Xiang and T. C. Green, "Analysis and Control of a Parallel DC Collection System for Wind Turbines with Single Active Bridge Converters," IEEE Energy Conversion Congress and Exposition (ECCE), Portland, OR, 2018, pp. 1005-1012, 2018.

[6] S. Galeshi, D. Frey, and Y. Lembeye, "Design Procedure of DC-DC Multi-Port Active-Bridge Converters," PCIM conference, 2020, in press.

[7] A. Rolán, F. Córcoles López, S. Bogarra, L. Monjo and J. Pedra, "Reduced-Order Models of Squirrel-Cage Induction Generators for Fixed-Speed Wind Turbines Under Unbalanced Grid Conditions," IEEE Transactions on Energy Conversion, vol. 31, pp. 566-577, June 2016.

[8] Q. Hao, Z. Li, F. Gao and J. Zhang, "Reduced-Order Small-Signal Models of Modular Multilevel Converter and MMC-Based HVdc Grid," IEEE Transactions on Industrial Electronics, vol. 66, pp. 22572268, March 2019.

[9] V. Purba, B. B. Johnson, M. Rodriguez, S. Jafarpour, F. Bullo and S. V. Dhople, "Reduced-order Aggregate Model for Parallel-connected 
Single-phase Inverters," IEEE Transactions on Energy Conversion, vol. 34, pp. 824-837, June 2019.

[10] K. Venkatachalam, C. R. Sullivan, T. Abdallah and H. Tacca, "Accurate prediction of ferrite core loss with nonsinusoidal waveforms using only Steinmetz parameters," in 2002 IEEE Workshop on Computers in Power Electronics, 2002. 